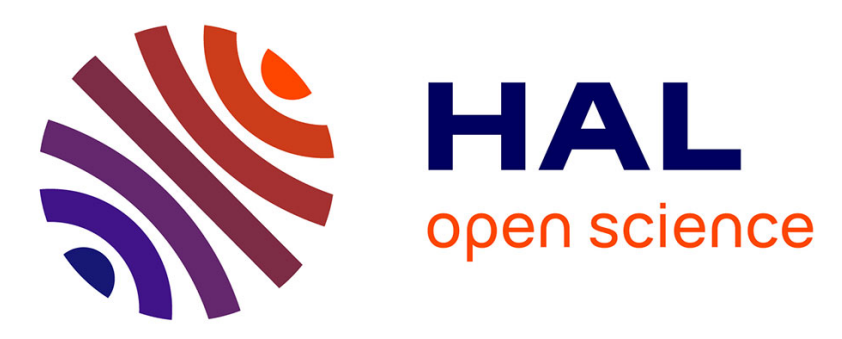

\title{
A New Metric for Statistical Analysis of Rigid Transformations: Application to the Rib Cage
}

Baptiste Moreau, Benjamin Gilles, Erwan Jolivet, Philippe Petit, Gérard

Subsol

\section{- To cite this version:}

Baptiste Moreau, Benjamin Gilles, Erwan Jolivet, Philippe Petit, Gérard Subsol. A New Metric for Statistical Analysis of Rigid Transformations: Application to the Rib Cage. GRAIL-MICGen-MFCA, Sep 2017, Québec City, Canada. pp.114-124, 10.1007/978-3-319-67675-3_11 . hal-01588289

\section{HAL Id: hal-01588289 \\ https://hal.science/hal-01588289}

Submitted on 6 Jul 2019

HAL is a multi-disciplinary open access archive for the deposit and dissemination of scientific research documents, whether they are published or not. The documents may come from teaching and research institutions in France or abroad, or from public or private research centers.
L'archive ouverte pluridisciplinaire HAL, est destinée au dépôt et à la diffusion de documents scientifiques de niveau recherche, publiés ou non, émanant des établissements d'enseignement et de recherche français ou étrangers, des laboratoires publics ou privés. 


\title{
A New Metric for Statistical Analysis of Rigid Transformations: Application to the Rib Cage
}

\author{
Baptiste Moreau ${ }^{1,2}$, Benjamin Gilles ${ }^{1}$, Erwan Jolivet ${ }^{3}$, Philippe Petit ${ }^{2}$, and \\ Gérard Subsol ${ }^{1}$ \\ 1 Research-team ICAR, LIRMM, University of Montpellier / CNRS, France \\ 2 LAB PSA Renault, France \\ ${ }^{3}$ CEESAR, Nanterre, France
}

\begin{abstract}
Statistical analysis of an anatomical structure composed of multiple objects is useful for many computational anatomy tasks as registration or classification. As rigid transformations do not belong to an Euclidean space, conventional mean and covariance formulas could not be applied to study the movement of each object with respect to the others. Some tools from Riemannian geometry are used instead, requiring the definition of a metric. We show that common metrics are not intuitive in the case of an object with an elongated shape and we propose a new one based on displacements of all the points of the structure. We describe the method to study the pose variability of a multi-object structure with this new metric. It is then applied to the statistical analysis of the rib cage which is composed of 24 elongated bones.
\end{abstract}

Keywords: Computational Anatomy, Rigid Transformations, Metric, Statistical Analysis, Rib Cage

\section{Introduction}

Statistical Shape Models are widely used to study variations of shapes for 3D anatomical structures. A simple and generic model to represent shapes is to distribute a set of points across the surface of the anatomical structure. Cootes et al. [1] proposed a method called Point Distribution Model, which consists of extracting the mean shape and a set of orthogonal modes of variation from a collection of training samples. Those statistical models are built on point-topoint correspondences. The number of variables could reach several hundreds depending on the point density of the geometry description. Such a statistical model correctly describes the intrinsic shape of the anatomical structure, and they are used to improve the performances of segmentation and registration algorithms [2], [3].

However, they cannot represent in an intuitive way an articulated anatomical structure which is composed of several substructures which can move relatively to the others, as for example a rib cage which is built from independent 24 ribs. In this case, we would like to characterize the intrinsic shapes of each substructure (e.g. each rib) but also the rigid motion (also called the pose) of the substructure 
with respect to a general frame (e.g. fixed on the spine). Capturing the pose variation of the substructures will lead to a Statistical Shape Model which is much more intuitively interpretable.

In general, a Statistical Shape Model is composed of a mean description and an analysis of the covariance of the variability of the structure shape with regard to the mean description which is given as a set of ordered orthogonal variation modes. In the case of sets of points, we are in an Euclidean space and we can use Principal Component Analysis (PCA). But, if we want to study pose statistics, we face the difficulty that the space of rigid transformations is not an Euclidean space. Thus, it is not possible to compute the mean of $3 \mathrm{D}$ rotations by using the conventional definition of the mean, regardless of the chosen representation for rotation (rotation matrix, rotation axis, Euler angles or quaternions).

Nevertheless, the set of rigid transformations $S E(3)$ has the structure of a manifold. A Riemannian metric on a manifold allows one to measure distances and angles. By defining a distance function between two 3D rigid transformations, the manifold locally resembles an Euclidean space, called the tangent space. Pennec [4] gave basic tools for probabilities and statistics in this general framework of Riemannian manifold and applies it to the statistics of rigid transformations. Fletcher [5] used the Riemannian framework to define Principal Geodesic Analysis (PGA) for statistical analysis of 3D boundary representations based on medial atoms linked by rigid transformations.

PGA aims at finding a geodesic subspace that minimize the sum of square distance of the points to their projection. However, as it is generally computationally complex, it is often approximated by a tangent PCA (tPCA), which maximizes the explanation of the covariance matrix by unfolding the distribution around the mean and making a standard PCA in the tangent space. Nevertheless, maximizing the explained variance (tPCA) and minimizing the unexplained variance (PGA) lead to different results on manifolds [6].

A key-point is then to define an efficient metric which is adapted to the application. In the case of the rib cage, we have very elongated objects: a small rotation applied on a point located far from the axis of rotation induces a large displacement even if the rotation angle is small. In this paper, we propose a new metric which takes into account in a more intuitive way the application of $3 \mathrm{D}$ rigid transformations on elongated objects. Then we use it to build an articulated Statistical Shape Model of the rib cage.

\section{Defining an Adapted Metric for Elongated Structures}

\subsection{Limits of the Current Metric}

Defining a distance function between two rigid transformations $H_{1}$ and $H_{2}$ is based on a norm function $N$ :

$$
d\left(H_{1}, H_{2}\right)=N\left(H_{2}^{-1} \circ H_{1}\right) .
$$

Several previous studies have defined a metric to perform tPCA on articulated anatomical structures with rigid transformations. First, a representation 
for $3 \mathrm{D}$ rotations has to be set: rotation matrix components [7], [3], rotation vector with the Rodrigues' formula [8], [9] or quaternions [10].

The most common norm is based on the vector representation of rotations. For $H \in S E(3)$ a rigid transformation decomposed into a rotation vector $\boldsymbol{r}=$ $\omega \cdot\left(n_{x}, n_{y}, n_{z}\right)^{T}$ and a translation $\boldsymbol{t}=\left(t_{x}, t_{y}, t_{z}\right)^{T}$ :

$$
N_{\text {rotv }}(H)^{2}=\boldsymbol{t}^{T} \cdot \boldsymbol{t}+\boldsymbol{r}^{T} \cdot \boldsymbol{r} .
$$

It is important to control the relative magnitude between translation (usually given in $\mathrm{mm}$ ) and rotation (usually in radians) by a normalization process. For example, Boisvert et al. [9] defined an empirical real number that controls the relative weight of the translation and rotation in the computation of the norm. Without this normalization process, the first modes would be translation modes as they account for most of the variance magnitude compared to the rotation which values in radians are generally quite low.

Another normalization consists of scaling the object geometry so that its mean radius is set to 1 . The displacements of points on the unit sphere is then given by the rotation angle in radian units [7].

With $\lambda$ a normalization factor, the norm function is then:

$$
N_{\text {rotv }}(H)^{2}=\boldsymbol{t}^{T} \cdot \boldsymbol{t}+\lambda \boldsymbol{r}^{T} \cdot \boldsymbol{r} .
$$

Nevertheless, to the authors' knowledge, available normalization methods cannot tackle the issue for elongated shape described below, as the ones shown in Figure 1.
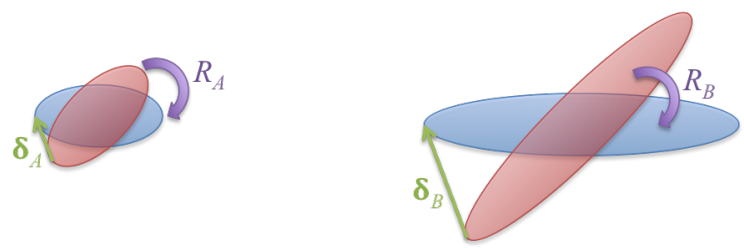

Fig. 1. Identical rotation applied on two objects: one compact and one elongated. The usual metric $N_{\text {rotv }}$ is the same in both cases $\left[R_{A}=R_{B} \Rightarrow N_{\text {rotv }}\left(R_{A}\right)=N_{\text {rotv }}\left(R_{B}\right)\right]$ although displacements are different $\left[\boldsymbol{\delta}_{\boldsymbol{A}}<\boldsymbol{\delta}_{\boldsymbol{B}}\right]$.

This paper aims at providing a generic metric for $S E(3)$, relevant for all kind of shapes including elongated shapes, in order to apply a tPCA. The second contribution is of a more practical value and lies in applying this technique to the construction of an articulated statistical shape model of the rib cage.

\subsection{A New Metric for Elongated Objects}

For an object, a rigid transformation $H \in S E(3)$ induces a displacement field $\boldsymbol{\delta}(H)$. The object is sampled by points and the vector $\boldsymbol{\delta}(H)$ contains the dis- 
placements of these points. The norm function is now defined as follow:

$$
N(H)^{2}=\boldsymbol{\delta}(H)^{T} \cdot \boldsymbol{\delta}(H) .
$$

This new norm can be compared with the common norm by introducing the moment of inertia.

The norm defined by rotation vector is commonly used [8], [9]. Let $\boldsymbol{r} \in S O(3)$ be a rotation vector. $\boldsymbol{r}=\omega \cdot \boldsymbol{n}$ with $\omega$ the angle of rotation and $\boldsymbol{n}$ the unit vector of rotation. We have seen that the usual norm is:

$$
N_{\text {rotv }}(\boldsymbol{r})^{2}=\boldsymbol{r}^{T} \cdot \boldsymbol{r}=\omega^{2} \cdot \boldsymbol{n}^{T} \cdot \boldsymbol{n} .
$$

The inertia tensor of the object is $\overline{\bar{I}}$. The moment of inertia is defined for the rotation as follows:

$$
I_{C}=\boldsymbol{n}^{T} \cdot \overline{\bar{I}} \cdot \boldsymbol{n} .
$$

For a point $\boldsymbol{p}_{\boldsymbol{k}}=(x, y, z)$ of the object, the displacement induced by the rotation $\boldsymbol{r}$ is $\boldsymbol{\delta}_{\boldsymbol{k}}=\boldsymbol{r} \times \boldsymbol{p}_{\boldsymbol{k}}$ with $\omega$ a small angle. With our metric:

$$
\begin{array}{r}
N(\boldsymbol{r})^{2}=\boldsymbol{\delta}_{\boldsymbol{k}}^{T} \cdot \boldsymbol{\delta}_{\boldsymbol{k}}, \\
=\omega^{2} \cdot \boldsymbol{n}^{T} \cdot\left(J_{k}^{T} \cdot J_{k}\right) \cdot \boldsymbol{n} \text { with } J_{k}=-\boldsymbol{p}_{\boldsymbol{k}} \times=\left(\begin{array}{ccc}
0 & z & -y \\
-z & 0 & x \\
y & -x & 0
\end{array}\right), \\
J_{k}^{T} \cdot J_{k}=\left(\begin{array}{ccc}
\left(y^{2}+z^{2}\right) & -x y & -x z \\
-y x & \left(z^{2}+x^{2}\right) & -y z \\
-z x & -z y & \left(x^{2}+y^{2}\right)
\end{array}\right) .
\end{array}
$$

Supposing that an object of mass $m$ is homogeneous and uniformly sampled with points $k$, the inertia tensor is:

$$
\overline{\bar{I}}=m \sum_{k} J_{k}^{T} . J_{k}
$$

Therefore, using the usual metric $N_{\text {rotv }}$ makes the assumption that the inertia tensor is the identity matrix, and therefore that the shape is spherical. This usual norm is a good approximation when the object of interest is compact, but with elongated shape like ribs, the norm should take into account the specific geometry. For this purpose, our method uses the inertia tensor inertia tensor. We believed that using this metric, distances between pose of arbitrary shaped objects will be more appropriately measured as there is no more requirement for ad-hoc normalization between rotations and translations.

Under certain conditions (uniformly sampled object centered on its center of mass, undergoing a rigid motion), our distance has a physical interpretation: it is proportional to the kinetic energy necessary to move the object from one position to another. 


\section{Statistical Description of Rigid Transformations}

\subsection{Pose Variations}

Some studies used a joint description to perform a tPCA [9], [11]. They defined an anatomical frame for each object and the transformation between two connected objects was a joint. In particular, the joint method for tPCA is suitable for the spine as it describes physical articulations, but there are drawbacks for other structures. First, the connection graph must not contain any cycles as it is the case for the rib cage (Figure 2). Indeed, a rib is articulated on both extremities with the spine and the sternum. Secondly, a fixed object as root of the tree must be determined. This can lead to numerical drift when an object is far from the fixed one. It is the same kind of issue than the elongated shape problem expressed previously.

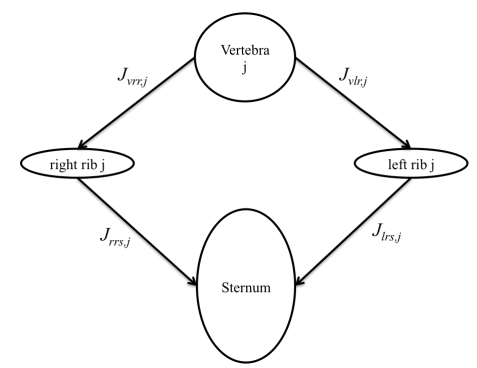

Fig. 2. Connection graph of the rib cage that shows the articulation loops.

To study pose variations of objects in the dataset, a generalized Procrustes analysis [12] is performed on each object separately. The rigid transformations (translation + rotation) applied to perform these multiple alignments as well as the mean shapes are used to study the pose variations (Figure 3). With this method, we go beyond issues described for the joint description.

For an object $j$ belonging to an instance $i, \boldsymbol{F}_{\boldsymbol{i}, \boldsymbol{j}}$ is the coordinate vector of points. The mean shape of an object $j$ among instances is $\overline{\boldsymbol{F}_{\boldsymbol{j}}}$ obtained from the multiple alignments. The rigid transformation $H_{i, j}$ is:

$$
H_{i, j}=\underset{H \in S E(3)}{\arg \min }\left\|H\left(\boldsymbol{F}_{\boldsymbol{i}, \boldsymbol{j}}\right)-\overline{\boldsymbol{F}_{\boldsymbol{j}}}\right\| .
$$

The rigid transformations obtained from the multiple alignments revealed how instances differ rigidly from the mean shape of each object.

The mathematical space of rigid transformations is not an Euclidean space. Therefore, conventional statistics do not apply. However, tPCA concepts can be applied to generalize statistical notions like average and variance [5]. 


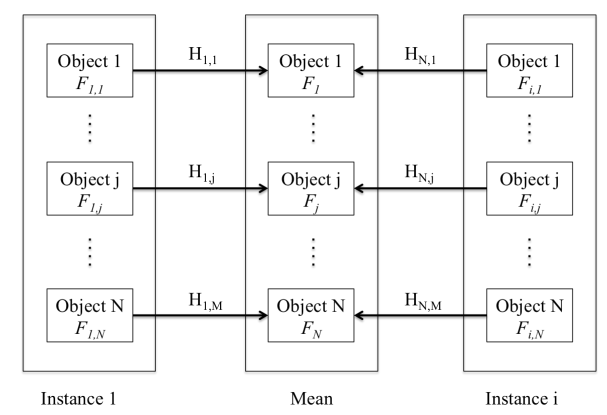

Fig. 3. The dataset contains instances composed of $\mathrm{N}$ objects described by point clouds $\boldsymbol{F}_{\boldsymbol{i}, \boldsymbol{j}} . H_{i, j}$ is the rigid transformation that aligns the instance $i$ on the mean shape.

\subsection{Generalized Covariance}

For a rigid transformation $H_{i, j} \in S E(3), \boldsymbol{\delta}_{\boldsymbol{j}}\left(H_{i, j}\right)=H_{i, j}\left(\overline{\boldsymbol{F}_{\boldsymbol{j}}}\right)-\overline{\boldsymbol{F}_{\boldsymbol{j}}}$ is the displacement field induced by $H_{i, j}$ on the mean shape. The norm function was defined as follow:

$$
N_{j}\left(H_{i, j}\right)^{2}=\boldsymbol{\delta}_{\boldsymbol{j}}\left(H_{i, j}\right)^{T} \cdot \boldsymbol{\delta}_{\boldsymbol{j}}\left(H_{i, j}\right) .
$$

The norm function is defined based on the mean shape, which means that only the mean shape inertia tensor is taken into account. This ensures that the norm does not change with the object being transformed.

With this definition of norm function, the distance between two rigid transformations, $H_{i_{1}, j}$ and $H_{i_{2}, j}$, from two different instances is:

$$
d\left(H_{i_{1}, j}, H_{i_{2}, j}\right)=N_{j}\left(H_{i_{2}, j}^{-1} \circ H_{i_{1}, j}\right) .
$$

This distance is left-invariant as $d\left(H_{3} \circ H_{1}, H_{3} \circ H_{2}\right)=d\left(H_{1}, H_{2}\right)$.

Let us call $\log$ the function that calculates the tangent description of a transformation according to the distance definition. The Exp function is the reverse of the $\log$ function.

$$
\begin{aligned}
\log _{\boldsymbol{I d}}\left(H_{i, j}\right) & =\boldsymbol{\delta}_{\boldsymbol{j}}\left(H_{i, j}\right), \\
\log _{\boldsymbol{H}_{\boldsymbol{i}_{1}, j}}\left(H_{i_{2}, j}\right) & =\boldsymbol{L o g}_{\boldsymbol{I} \boldsymbol{d}}\left(H_{i_{2}, j}^{-1} \circ H_{i_{1}, j}\right) . \\
\operatorname{Exp}_{I d}\left(\boldsymbol{\delta}_{\boldsymbol{i}, \boldsymbol{j}}\right) & =\underset{H \in S E(3)}{\arg \min }\left(N_{j}(H)^{2}\right), \\
\operatorname{Exp}_{H_{i_{1}, j}}\left(\boldsymbol{\delta}_{\boldsymbol{i}_{2}, \boldsymbol{j}}\right) & =H_{i_{1}, j} \circ \operatorname{Exp}_{I d}\left(\boldsymbol{\delta}_{\boldsymbol{i}_{\boldsymbol{2}}, \boldsymbol{j}}\right) .
\end{aligned}
$$

They correspond to the Riemannian Exp/Log for the left-invariant distance generated by the inertia tensor. This $L o g$ function is valid for small angles of rotation to get the property: $\boldsymbol{L o g}_{\boldsymbol{I} \boldsymbol{d}}\left(H^{-1}\right) \approx-\boldsymbol{L o g}_{\boldsymbol{I} \boldsymbol{d}}(H)$. 
A generalization of the usual mean - called Fréchet mean - can be obtained by defining for an object $j$ the mean as the rigid transformation $\mu_{j}$ that minimizes the sum of the distances with respect to the set of rigid transformations $H_{1, j}, \ldots, H_{N_{i}, j}$.

$$
\mu_{j}=\underset{H \in S E(3)}{\arg \min }\left(\sum_{i=1}^{N_{i}} d\left(H, H_{i, j}\right)^{2}\right) .
$$

The Fréchet mean is computed by a simple gradient descent procedure [4]. This procedure is summarized by the following recurrent equation, with $i$ an instance and $N_{i}$ the number of instances in the sample.

$$
\mu_{j, n+1}=\operatorname{Exp}_{\mu_{j, n}}\left(\frac{1}{N_{i}} \sum_{i=1}^{N_{i}} \boldsymbol{L}_{\boldsymbol{o g}} \boldsymbol{\mu}_{j, n}\left(H_{i, j}\right)\right) .
$$

In addition, a dispersion measure is needed to perform most tasks of practical interest. The covariance is usually defined as the expectation of the matrix product of the vectors from the mean. Thus, a similar definition for tPCA would be to compute the expectation in the tangent space of the mean using the $\log$ function. Let $j_{1}, j_{2}$ be two objects with two mean shapes $\overline{\boldsymbol{F}_{\boldsymbol{j}_{1}}}, \overline{\boldsymbol{F}_{\boldsymbol{j}_{2}}}$, two sets of rigid transformations $\operatorname{set}_{j_{1}}=\left\{H_{1, j_{1}}, \ldots, H_{N_{i}, j_{1}}\right\}$, set $_{j_{2}}=\left\{H_{1, j_{2}}, \ldots, H_{N_{i}, j_{2}}\right\}$ and two Fréchet means $\mu_{j_{1}}, \mu_{j_{2}}$, respectively. The definition of the covariance between these two objects is:

$$
\operatorname{Cov}\left(\operatorname{set}_{j_{1}}, \operatorname{set}_{j_{2}}\right)=\frac{1}{N_{i}-1} \sum_{i=1}^{N_{i}} \boldsymbol{L o g}_{\boldsymbol{\mu}_{j_{1}}}\left(H_{i, j_{1}}\right) \cdot \boldsymbol{L o g}_{\boldsymbol{\mu}_{\boldsymbol{j}_{\mathbf{2}}}}\left(H_{i, j_{2}}\right)^{T} .
$$

The displacement $\boldsymbol{L o g}_{\boldsymbol{\mu}_{j}}\left(H_{i, j}\right)$ is a vertical vector of size $3 N_{j}$ with $N_{j}$ the number of points of the object $j$.

Unlike the manifold itself, the tangent space is a vector space and its basis could be changed using a simple linear transformation. With the definition of covariance generalized, eigenvectors are computed with the tangent description, and the Exp function is applied to get principal components in terms of rigid transformations. This is the simplest generalization called tangent PCA (tPCA), which amounts to unfold the whole distribution in the tangent space at the mean, and to compute the principal components of the covariance matrix in the tangent space. tPCA is good for analyzing data which are sufficiently centered around a central value [13].

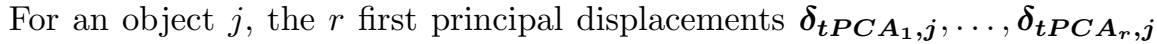
are obtained by tPCA. Associated scores $\alpha_{t P C A_{1}, i, j}, \ldots, \alpha_{t P C A_{r}, i, j}$ are describing the $i$-instance position along the principal displacements, with dataset variances (eigenvalues) $\lambda_{t P C A_{1}, j}, \ldots, \lambda_{t P C A_{r}, j}$. The following equation is used to re-create an instance's object with a reduced number of modes:

$$
\begin{aligned}
H_{t P C A, i, j} & =\operatorname{Exp}_{\mu_{j}}\left(\sum_{k=1}^{r} \alpha_{t P C A_{k}, i, j} . \boldsymbol{\delta}_{\boldsymbol{t P C A} \boldsymbol{A}_{\boldsymbol{k}}, \boldsymbol{j}}\right), \\
\boldsymbol{F}_{\boldsymbol{t P C A}, \boldsymbol{i}, \boldsymbol{j}} & =H_{t P C A, i, j}\left(\overline{\boldsymbol{F}_{\boldsymbol{j}}}\right) .
\end{aligned}
$$




\subsection{Application to the Rib Cage}

The dataset of rib cage meshes was obtained from 3D CT images. The 26 subjects were males, around 70 years old $(73.3 \pm 11)$ with a standard morphology $(70 \pm 9.8 \mathrm{~kg}$ in weight; $172 \pm 5.5 \mathrm{~cm}$ in height). Meshes were obtained by using a model-based segmentation described in [14]. This also ensures point correspondences between all the meshes. The atlas mesh $M_{\text {ref }}$ was constituted of 37 independent sub-meshes (1,570 nodes per mesh in average \pm 314$)$ representing the 12 vertebrae, 24 ribs and the sternum.
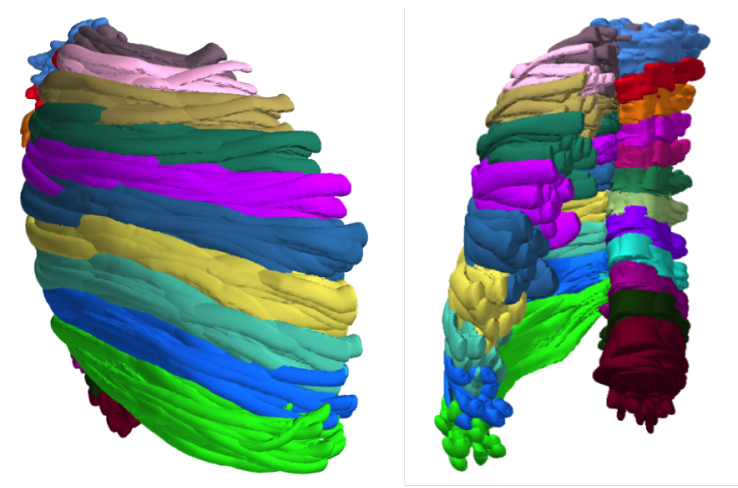

Fig. 4. Illustration of superposed right-side rib cages from the dataset with variations of pose and shape in lateral (left) and frontal views (right). The various colors enable to distinguish bones.

We applied the proposed method to the rib cage dataset, instances were subjects and objects were bones. A pre-alignment was applied to superimpose subjects (Figure 4). Multiple alignments were applied to compute rigid transformations used in tPCA and mean shapes used to define the norm.

To illustrate (Figure 5) the different orthogonal modes retrieved using the proposed method, three models were reconstructed for each of the first three principal modes. Those models were reconstructed by setting $\alpha_{m}$ to $-3 \sqrt{\lambda_{m}}, 0,3 \sqrt{\lambda_{m}}$ for $m=1,2,3$ in the formula:

$$
\begin{aligned}
& H_{t P C A_{m}, j}=\operatorname{Exp}_{\mu_{j}}\left(\alpha_{m} \cdot \boldsymbol{\delta}_{\boldsymbol{t P C A} \boldsymbol{A}_{\boldsymbol{m}}, \boldsymbol{j}}\right), \\
& \boldsymbol{F}_{\boldsymbol{t P C A _ { m }}, \boldsymbol{j}}=H_{t P C A_{m}, j}\left(\overline{\boldsymbol{F}_{\boldsymbol{j}}}\right) \text {. }
\end{aligned}
$$

As expected, a relatively small number of modes accounted for most of the pose variability in the dataset. The accuracy of the model was evaluated by reconstructing subjects from a reduced number of components and comparing them with true geometries. The reconstruction errors were calculated as mean Euclidean distances.

The method to study pose variations was compared with the method using rotation vector description as tangent space [8], [9]. The reconstruction distances 

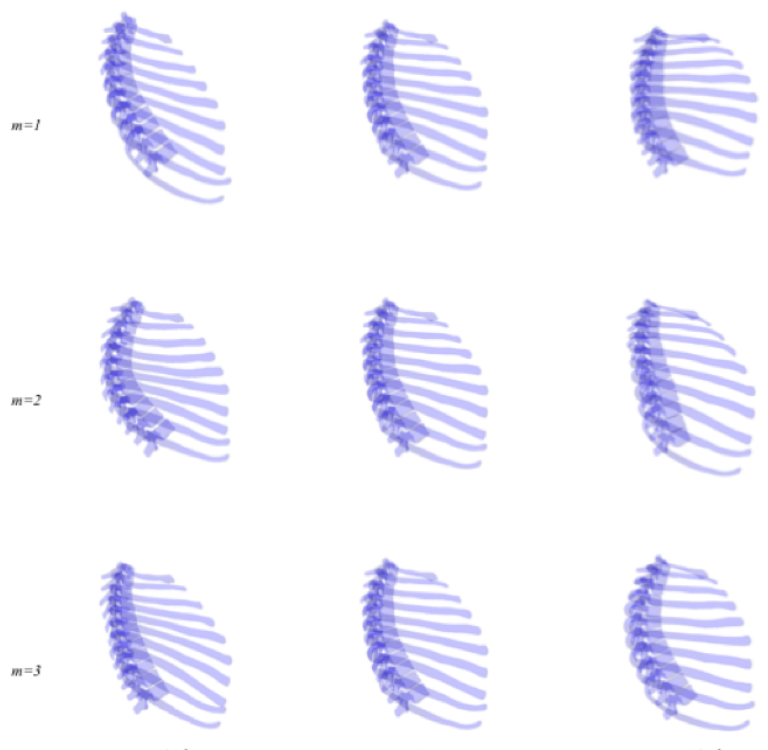

$\alpha_{m}=0$

$\alpha_{m}=-3 \lambda_{m}^{-1}$
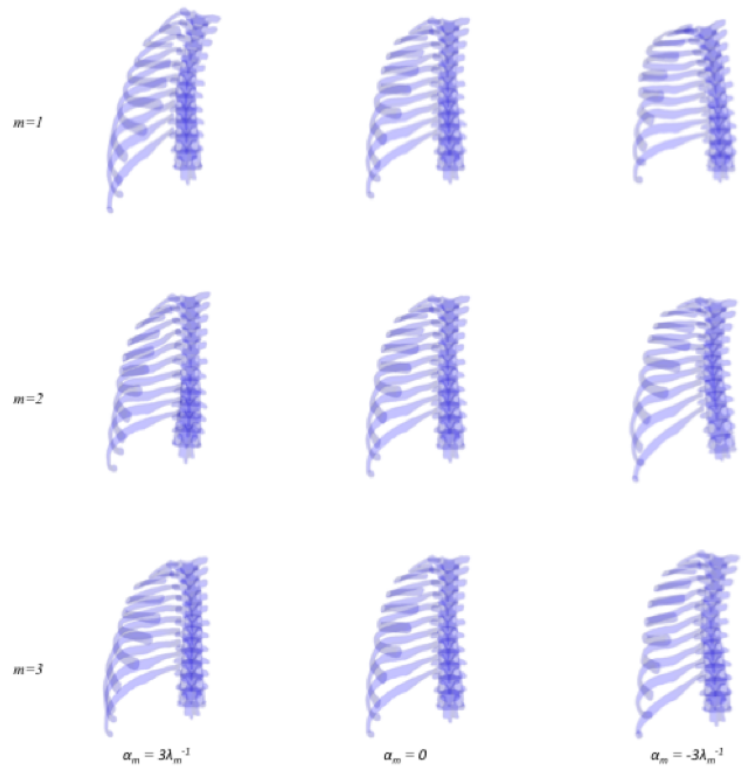

Fig. 5. First $(m=1)$, second $(m=2)$ and third $(m=3)$ principal modes of the rib cages dataset in lateral view and frontal view. The right side fo the rib cage models was rendered for $-3,0,3$ times the standard deviation explained by the corresponding mode. The middle column is the mean shape. 
were better in average for a reduced number of tPCA modes with the method presented in this study. We can see in Figure 6 on the left graph that for a small number of modes, the error is much lower. The proposed metric facilitated lower reconstruction error for given number of components retained. About 4 components are needed for a similar reconstruction error obtained with 10 components of the standard metric. This shows that the new method is adapted to study articulated elongated bones like ribs.
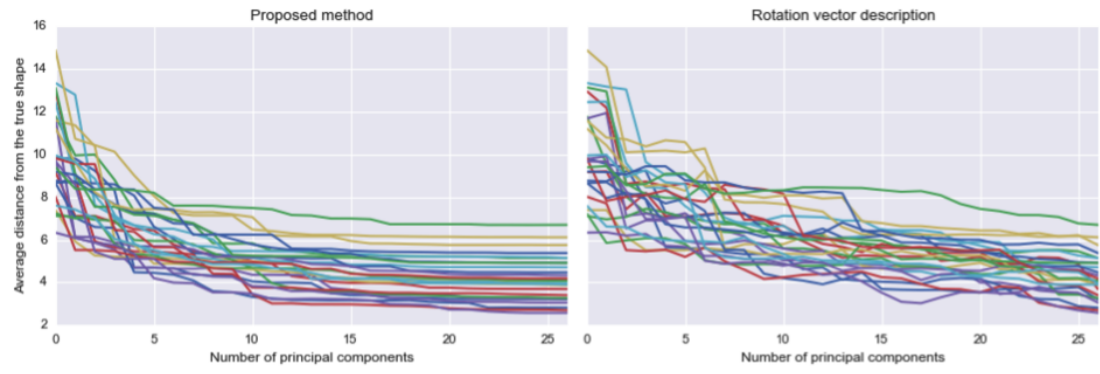

Fig. 6. Subject reconstruction error with respect to the number of principal modes after a tPCA. Each color refers to a subject. Left, principal modes were computed with the new metric. Right, principal modes were obtained with the standard rotation metric.

\section{Conclusion}

In this paper, we introduced a new metric to perform statistical analysis on rigid transformations. Unlike previous metric, this one takes into account the shape of the object by integrating the inertia matrix. A tPCA was performed with this new metric on a dataset of rib cages. As the rib is an elongated anisotropic structure which is very elongated, it highlights the interest of this metric. In particular, the principal modes we obtained appear more suitable to reconstruct a subject with a reduced number of parameters.

\section{References}

1. Cootes, T.F., Taylor, C.J., Cooper, D.H., Graham, J.: Active Shape Models-Their Training and Application. Computer Vision and Image Understanding 61(1) (January 1995) 38-59

2. Zheng, G., Gollmer, S., Schumann, S., Dong, X., Feilkas, T., Ballester, M.A.G.: A 2D/3D correspondence building method for reconstruction of a patient-specific 3D bone surface model using point distribution models and calibrated X-ray images. Medical image analysis 13(6) (2009) 883-899 
3. Rasoulian, A., Rohling, R., Abolmaesumi, P.: Lumbar spine segmentation using a statistical multi-vertebrae anatomical shape+pose model. IEEE transactions on medical imaging 32(10) (2013) 1890-1900

4. Pennec, X.: Intrinsic statistics on Riemannian manifolds: Basic tools for geometric measurements. Journal of Mathematical Imaging and Vision 25(1) (2006) 127-154

5. Fletcher, P.T., Lu, C., Pizer, S.M., Joshi, S.: Principal geodesic analysis for the study of nonlinear statistics of shape. IEEE Transactions on Medical Imaging 23(8) (2004) 995-1005

6. Sommer, S., Lauze, F., Nielsen, M.: Optimization over geodesics for exact principal geodesic analysis. Advances in Computational Mathematics 40(2) (2014) 283-313

7. Bossa, M.N., Olmos, S.: Statistical model of similarity transformations: Building a multi-object pose. In: 2006 Conference on Computer Vision and Pattern Recognition Workshop (CVPRW'06), IEEE (2006) 59-59

8. Anas, E.M.A., Rasoulian, A., John, P.S., Pichora, D., Rohling, R., Abolmaesumi, P.: A statistical shape+ pose model for segmentation of wrist CT images. In: SPIE Medical Imaging, International Society for Optics and Photonics (2014) 90340T90340T

9. Boisvert, J., Cheriet, F., Pennec, X., Labelle, H., Ayache, N.: Geometric variability of the scoliotic spine using statistics on articulated shape models. IEEE Transactions on Medical Imaging 27(4) (2008) 557-568

10. Said, S., Courty, N., Le Bihan, N., Sangwine, S.J.: Exact principal geodesic analysis for data on so (3). In: Signal Processing Conference, 2007 15th European, IEEE (2007) 1701-1705

11. Bindernagel, M., Kainmueller, D., Seim, H., Lamecker, H., Zachow, S., Hege, H.C.: An articulated statistical shape model of the human knee. In: Bildverarbeitung für die Medizin 2011. Springer (2011) 59-63

12. Goodall, C.: Procrustes methods in the statistical analysis of shape. Journal of the Royal Statistical Society. Series B (Methodological) (1991) 285-339

13. Pennec, X.: Barycentric subspaces and affine spans in manifolds. In: International Conference on Networked Geometric Science of Information, LNCS, volume 9389, Springer (2015) 12-21

14. Gilles, B., Revéret, L., Pai, D.K.: Creating and Animating Subject-Specific Anatomical Models. Computer Graphics Forum 29(8) (December 2010) 23402351 